\title{
Liver and Intrahepatic Bile Duct Neoplasm
}

National Cancer Institute

\section{Source}

National Cancer Institute. Liver and Intrahepatic Bile Duct Neoplasm. NCI Thesaurus.

Code C7103.

A benign or malignant neoplasm that affects the liver parenchyma or intrahepatic bile ducts. Representative examples of benign neoplasms include hepatocellular adenoma, and bile duct adenoma. Representative examples of malignant neoplasms include hepatocellular carcinoma, intrahepatic cholangiocarcinoma, and lymphoma. 